\title{
Sakurai's Object, V605 Aql and FG Sge: An Evolutionary Sequence Revealed
}

\author{
Timothy M. Lawlor \\ Pennsylvania State University - Wilkes Barre, Lehman, PA 18627, USA
}

James MacDonald

University of Delaware, Newark, DE 19716, USA

\begin{abstract}
We have completed a grid of stellar evolution calculations to study the behavior of the born again phenomenon. All our evolutionary sequences begin with a uniform composition $1 \mathrm{M}_{\odot}$ star on the pre-main sequence Hayashi phase and end on the white dwarf cooling track. We find a very late thermal pulse occurs in $10-15$ percent of cases. Our models supply an answer to the question of why the born again stars V4334 Sgr (Sakurai's Object) and V605 Aql have a significantly shorter evolutionary time scale than the otherwise similar born again star FG Sge. Models with low convective mixing efficiency, $\eta=10^{-4}$, first evolve quickly to the AGB, return to the blue, and then evolve more slowly back to the AGB for a second time before finally returning to the white dwarf cooling track. The difference in evolution time scales can then be explained by proposing that Sakurai's Object is evolving to the AGB for the first time but FG Sge has been observed during its second return to the AGB.
\end{abstract}

\section{Introduction}

Stars of initial mass $\leq 10.5 \mathrm{M}_{\odot}$ evolve onto the asymptotic giant branch (AGB) after completing core helium burning (Iben \& MacDonald 1995; Ritossa, GarcíaBerro, \& Iben 1999; Schwarzschild \& Härm 1965). On the AGB, the star undergoes a series of thermal helium pulses and loses mass at high rates, $10^{-7}$ to $10^{-5} \mathrm{M}_{\odot} \mathrm{yr}^{-1}$. When the mass of the hydrogen-rich envelope drops below a critical value $\left(\sim 10^{-3} \mathrm{M}_{\odot}\right)$, the envelope begins a phase of contraction at constant luminosity. During the contraction the effective temperature increases. When thermonuclear reactions cease, the star begins to cool and moves in the HR-diagram to the white dwarf cooling track. However, for stars entering the cooling track with enough helium remaining, a final helium shell flash can occur (Schönberner 1979; Iben 1982). As a result of this very late thermal pulse (VLTP), the star returns to the AGB. The transition is characterized by an increase in luminosity and a drop in effective temperature in a very short time ( $\sim 5$ to 15 years). Additionally, the stellar photosphere becomes deficient in hydrogen, rich in helium, and enriched in C, N, O, and s-process materials. 
The code used is a modified version of that developed by Eggleton (1971, 1972). We use a prescription for the diffusion coefficient that is consistent with mixing length theory. The diffusion coefficient is $\sigma_{c o n}=\beta w_{\text {con }} l$, where $w_{c o n}$ is the convective velocity, $l$ is the mixing length, and $\beta$ is a dimensionless convective efficiency parameter. For details of the code see Lawlor \& MacDonald (2002). Mass loss is included by using a scaled Reimers (1975) mass loss law, for cool stars $\left(T_{\text {eff }} \leq 10^{4} \mathrm{~K}\right)$, and an approximation to the theoretical result of Abbott (1982) for hot stars $\left(T_{\text {eff }} \geq 10^{4} \mathrm{~K}\right)$.

We have evolved $1 \mathrm{M}_{\odot}$ stars of metallicity $Z=0.001,0.004,0.01$ and 0.02 from the pre-main sequence Hayashi phase to the white dwarf cooling track. Based on the findings of Jimenez et al. (1995) and others, we adopt a value for the mass loss efficiency parameter. To generate a grid of models suitable for investigation of the VLTP, we vary the mass loss rate starting from the peak of the last AGB thermal pulse by choosing different values for the mass loss parameter, $\eta$. This gives a complete range of the possible helium layer masses at the point when the star leaves the asymptotic giant branch.

\section{Mixing Efficiency and Dependence on $Z$}

Herwig (2001) has shown that the evolutionary time scale of VLTP stars strongly depends on the value chosen for the convective mixing efficiency parameter $\eta$. To explore the dependence of our results on this parameter we have calculated for the $Z=0.02, \eta=0.06$ case evolutionary sequences with $\beta=10^{-4}, 10^{-3}$ and $10^{-2}$. In Fig. 1 we show an HR-diagram contrasting VLTP models of differing mixing efficiency parameters. The evolutionary paths shown have a double loop

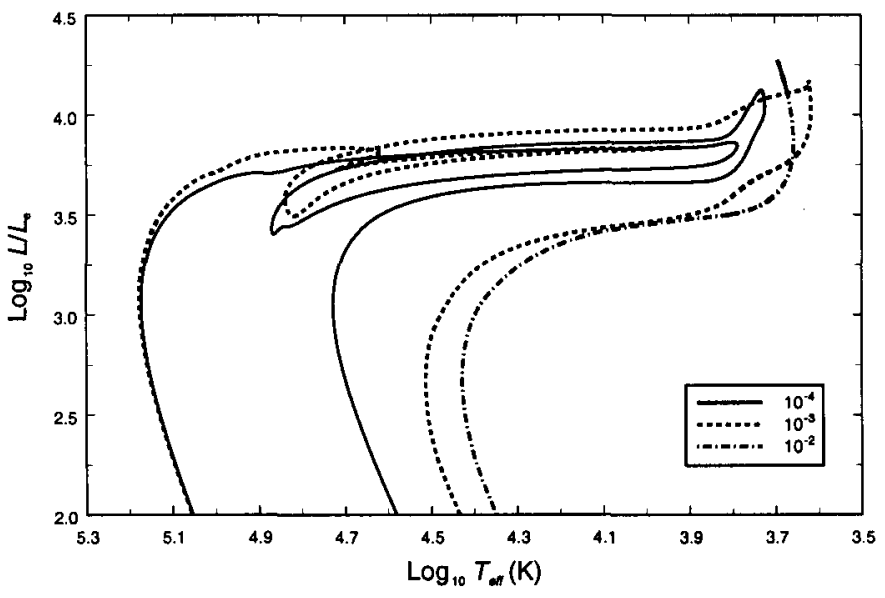

Figure 1. Dependance of the HR-diagram of VLTP models on the convective efficiency parameter, $\beta$ 
structure. The double loop in the HRD is a result of the penetration of the He-flash driven convection zone into $\mathrm{H}$-rich layers. When protons are convected to hot enough regions they are captured by ${ }^{12} \mathrm{C}$ nuclei. Ingestion of protons by the convection zone continues until the rate of energy generation by proton captures becomes comparable to that from helium burning. The entropy produced by proton captures forces the splitting of the convective zone into two distinct convective zones: one powered by hydrogen burning and the other by helium burning (Iben \& MacDonald 1995). The initial expansion of the envelope is powered by the H-burning shell. When this dies out, the envelope initially contracts. The second expansion of the envelope is powered by the continuing He-burning shell. As this dies out, the envelope contracts again and the star returns to the white dwarf domain. For $\beta=10^{-4}$, mixing is relatively weak and composition gradients remain in the envelope throughout much of the evolution, even when the envelope is fully convective. When the star reaches red giant dimensions for the first time after the VLTP, the mass fractions at the photosphere are $X=0.35, Y=0.48, Z_{\mathrm{C}}=0.12$ and $Z_{\mathrm{O}}=0.04$. For $\beta=10^{-3}$ evolution initially occurs at a rate similar to the case with $\beta=10^{-4}$ but the evolution after the first return to the blue is much quicker. The time from the second maximum in temperature to the second minimum is approximately 25 years compared to 500 years for the case with $\beta=10^{-4}$.

We attribute the shorter lifetime of the second giant phase to the greater mean molecular weight in the envelope that results from the higher convective efficiency. The structure of the star is essentially a degenerate CO core with a helium burning shell and a hydrogen depleted envelope. For the core masses of our models such stars do not become red giants (Weiss 1987). For $\beta=10^{-2}$, the initial evolution is similar to that of $\beta=10^{-3}$. However, the even greater convective efficiency causes complete homogenization of the envelope on the first return to the giant branch. The envelope abundances at this stage are $X=0.04, Y=0.55, Z_{\mathrm{C}}=0.28$ and $Z_{\mathrm{O}}=0.08$. The densities in this region are low enough that convection is not completely efficient. The radiation pressure gradient is more than enough to balance gravity. Hence our assumption of hydrostatic equilibrium requires that the gas pressure gradient be opposite to that of the radiation pressure. This causes a density inversion. As the density decreases convection becomes less efficient and this causes a steeper radiation pressure gradient that in turn requires a steeper gas pressure gradient to maintain hydrostatic equilibrium. This leads to an unstable situation that prevents continuation of the calculation. Because the time scales with lower are similar to the evolutionary time scales of FG Sge and SO, and because of the breakdown of the hydrostatic approximation after the first return to the AGB for $\beta=10^{-2}$, we choose $\beta=10^{-4}$ for the rest of the calculations. A representative HRD for a VLTP is shown in Fig. 2. In the top plot, we show the evolution from the end of the AGB to the white dwarf cooling track. The details of the double loop evolution are shown in the bottom plot. The times between the labeled points are given in Table 1 for models of different metallicities. During the double loop, extremely rapid and sweeping changes in surface chemical composition also take place. Over this short period, the surface of the star becomes deficient in hydrogen and rich in helium. It also becomes enriched in carbon, oxygen, and nitrogen. In Table 2 we compare, in mass fractions, the amount of $\mathrm{H}, \mathrm{He}, \mathrm{C}, \mathrm{O}$, 

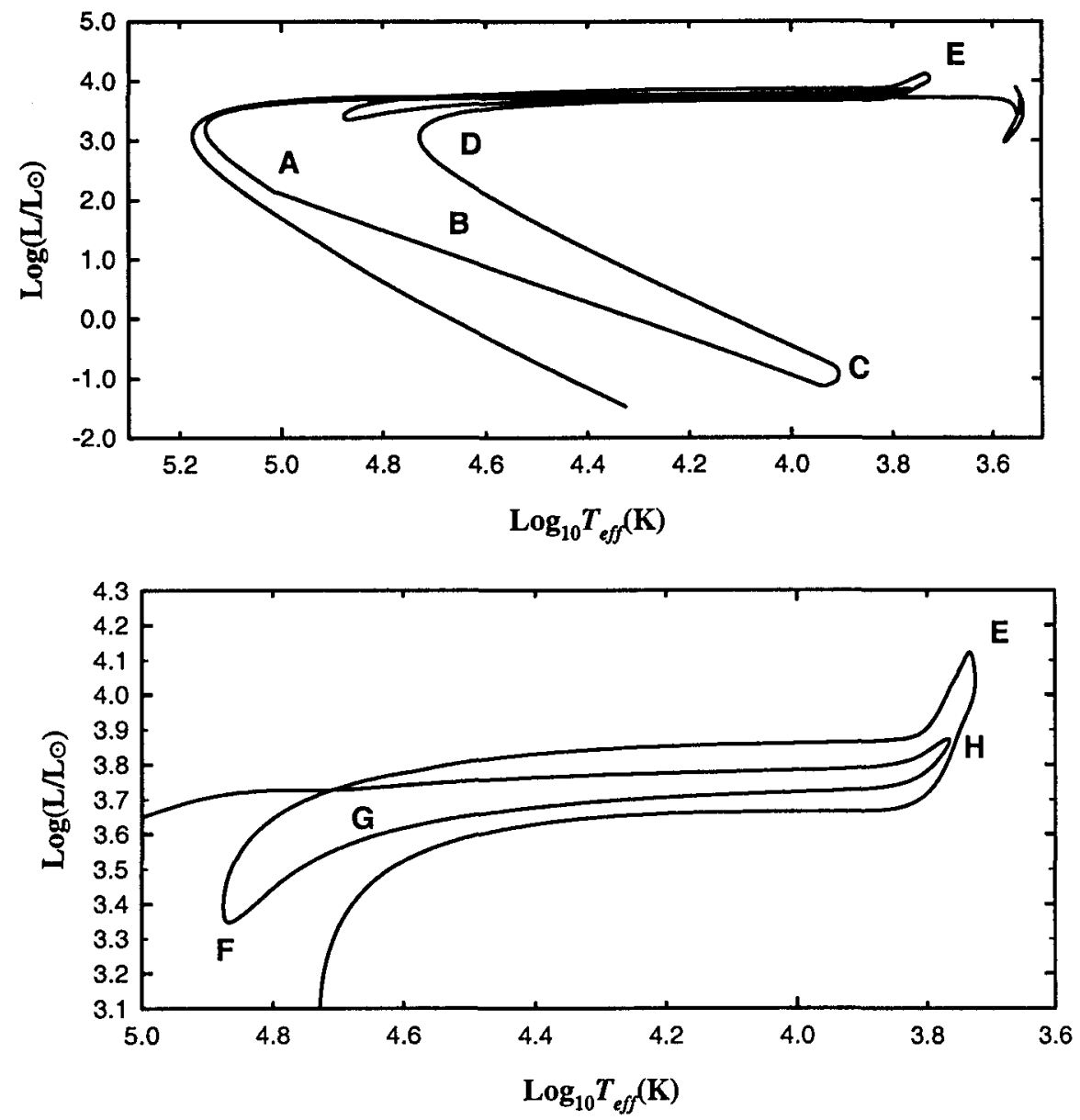

Figure 2. Hertzsprung-Russell diagram for a VLTP.

and $\mathrm{N}$ before and after a very late helium flash. Also compared is the change in $T_{\text {eff }}$, luminosity, and $\log R$.

\section{Observed VLTP Objects - the Time Scale Problem}

Three directly observed objects that exhibit behavior consistent with the born again scenario are Sakurai's Object (V4334 Sgr), FG Sge, and V605 Aql. Sakurai's Object (SO) was discovered in 1996 as a star of 11th magnitude. Prediscovery observations show that it began to brighten in late 1994 or early 1995 . It has increased in visual luminosity by as much as four magnitudes and decreased significantly in temperature within the last six years. FG Sge has been continuously observed to be brightening and cooling since the late $1800 \mathrm{~s}$, and V605 Aql is believed to have flashed in about 1917, reaching its peak only two 
Table 1. Time scales for the VLTP (in years)

\begin{tabular}{cccccccc}
\hline metallicity $(\mathrm{Z})$ & $\mathrm{A}-\mathrm{B}$ & $\mathrm{B}-\mathrm{C}$ & $\mathrm{C}-\mathrm{D}$ & $\mathrm{D}-\mathrm{E}$ & $\mathrm{E}-\mathrm{F}$ & $\mathrm{F}-\mathrm{G}$ & $\mathrm{G}-\mathrm{H}$ \\
\hline \hline$Z=0.001$ & 6100 & 0.19 & 0.16 & 4.2 & 16 & 125 & 175 \\
$Z=0.004$ & 14000 & 0.032 & 0.10 & 6.1 & 19 & 150 & 250 \\
$Z=0.010$ & 1100 & 0.065 & 0.18 & 9.5 & 31 & 240 & 280 \\
$Z=0.020$ & 5900 & 0.12 & 0.25 & 12 & 33 & 290 & 250 \\
\hline
\end{tabular}

Table 2. Surface parameters before and after the VLTP (given at points, $\mathrm{A}$ and $\mathrm{H}$, shown in Fig. 2)

\begin{tabular}{l|llll|llll}
\hline & $(\mathrm{A})$ & & & & & $(\mathrm{H})$ & & \\
\hline $\mathrm{Z}$ & 0.001 & 0.004 & 0.01 & 0.02 & 0.001 & 0.004 & 0.01 & 0.02 \\
$\mathrm{H}$ & 0.70 & 0.71 & 0.70 & 0.68 & 0.14 & 0.40 & 0.44 & 0.51 \\
$\mathrm{He}$ & 0.287 & 0.281 & 0.29 & 0.30 & 0.73 & 0.50 & 0.44 & 0.40 \\
$\mathrm{C}$ & 0.0094 & 0.004 & 0.002 & 0.003 & 0.084 & 0.064 & 0.077 & 0.055 \\
$\mathrm{~N}$ & 0.0004 & 0.0005 & 0.001 & 0.002 & 0.015 & 0.006 & 0.004 & 0.005 \\
$\mathrm{O}$ & 0.003 & 0.003 & 0.005 & 0.01 & 0.023 & 0.026 & 0.019 & 0.02 \\
metals & 0.001 & 0.0014 & 0.003 & 0.005 & 0.01 & 0.006 & 0.008 & 0.004 \\
$\log T_{\text {eff }}$ & 5.06 & 5.02 & 5.01 & 5.01 & 4.49 & 3.80 & 3.75 & 3.77 \\
$\log L / L_{\odot}$ & 2.00 & 1.92 & 2.08 & 2.13 & 4.11 & 4.03 & 3.94 & 3.87 \\
$\log R(\mathrm{~cm})$ & 9.25 & 9.29 & 9.38 & 9.41 & 11.43 & 12.78 & 12.83 & 12.77 \\
\hline
\end{tabular}

years later. All three objects have increased in luminosity, decreased in temperature, and experienced significant changes in chemical composition. With this theoretical picture of very late helium flash behavior for low convective efficiency, $\beta=10^{-4}$, we are poised to answer an important and previously troubling question: Why have SO and V605 Aql been observed to evolve to cool temperatures in about 2 to 6 years, while FG Sge has clearly been observed to be cooling for some 120 years? In Fig. 3 we compare how radii change as a function of time for SO, FG Sge, V605 Aql, and an evolutionary model. This comparison shows clearly that a possible solution to the time scale problem is that SO and FG Sge are at different stages of a common very late flash evolution. Though the observations for SO are sparse, the slope of the curve resembles closely the first and much faster ( 4.5 to 8.5 years) approach to cooler temperatures. The slope, shape, and time scale for FG Sge unmistakably resembles the second, slower (200 to 550 years) approach to cooler temperatures. If this explanation of the difference in evolutionary time scales of the objects is correct, we can predict that $\mathrm{SO}$ will increase in temperature (along the top of the loop in the HR diagram) in a slightly slower time scale (20 to 50 years) than it decreased. Following this increase it would again brighten and cool gradually (200 to 500 years), in the same fashion as has been observed for FG Sge.

It is expected that it will spend approximately 50 to 100 years on the warmer side of the loop before making this final approach to the AGB. Though the data for V605 Aql are incomplete, they add important evidence. We can say with confidence that it underwent a flash in 1917, that it cooled and grew to giant size on roughly the same time scale as $\mathrm{SO}$, and recent observations indicate that the central star is about the same luminosity and has an effective temperature $T_{\text {eff }} \geq 50,000 \mathrm{~K}$. Because its minimum temperature had been reached 83 years 


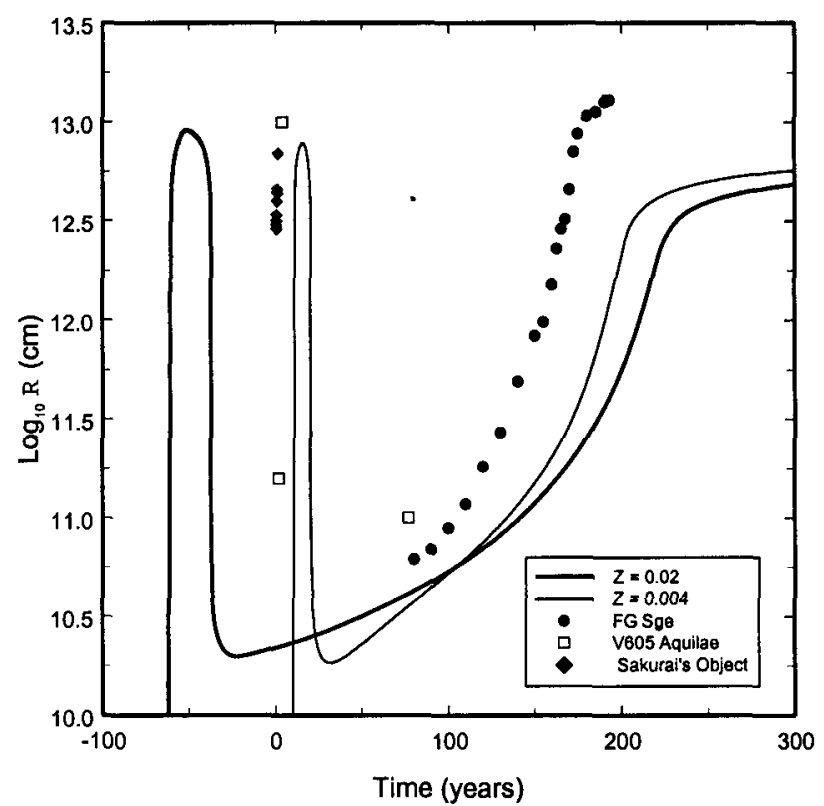

Figure 3. A comparison of radius estimates from the observations of FG Sge, V605 Aquilae and Sakurai's Object with model radii as a function of time.

ago, V605 Aql provides a single and perfect example of a link between SO and FG Sge. Specifically, our models evolve from cooler to warmer temperatures at roughly constant luminosity (dropping about half an order of magnitude) and shrinking to approximately solar size with a time scale between 20 and 50 years (depending on metallicity), as is the case for V605 Aql. Each of the three observed objects represents one of the three crossings of the HR diagram: from hot to cool (SO); from cool to hot (V605 Aql); and then back to a cool giant (FG Sge).

\section{Conclusions}

We present the results of calculations of the evolution of $1 \mathrm{M} \odot$ stars relevant to the investigation of the VLTP. We find that the light curves of SO, V605 Aql, and FG Sge can be explained in a single model in which the convective mixing efficiency is substantially reduced below that obtained from standard MLT. In this case, the born again stars follow a double loop path in the HR Diagram in which they first evolve quickly to the AGB, return to the blue, and then evolve more slowly back to the AGB before finally returning to the WD cooling track. The observed time scale differences result from SO having evolved to the AGB for the first time whereas FG Sge was observed during its second return to the AGB. A critical aspect of the double loop evolution is the requirement 
that the convective mixing efficiency be much lower than predicted by standard mixing length theory. Too efficient mixing leads to a completely homogenized envelope with a very low hydrogen abundance. A giant configuration is then not possible for our stars with low core masses and the second expansion to red giant dimensions does not occur. Our low convective mixing efficiency model allows us to make some testable predictions for the future evolution each of the three objects. Clayton \& De Marco (1997) have suggested that V605 Aql may be a glimpse into the future of SO. Our calculations supports that prediction and also indicate that FG Sge is showing us the future of both SO and V605 Aql. Specifically, we expect SO to move to warmer temperatures in the next 20 to 50 years, and that it then will resemble V605 Aql's present state (and this should be soon observable), and we expect V605 Aql to cool back toward the AGB but not noticeably for as much as 50 to 70 years, at which time it will evolve in the same way as has been observed for FG Sge for the last 120 years. Finally, FG Sge will show signs of increasing effective temperature by about 1,500 to $2,000 \mathrm{~K}$ in as soon as 10 to 20 years.

\section{References}

Abbott, D. 1982, ApJ, 259, 282

Clayton, G. C., \& De Marco, O. 1997, AJ, 114, 2679

Eggleton, P. P. 1971, MNRAS, 151, 351

Eggleton, P. P. 1972, MNRAS, 156, 361

Herwig, F. 2001, ApJ, 554, L71

Iben, I. 1982, ApJ, 260, 821

Iben, I., \& MacDonald, J. 1995, in White Dwarfs, Proceedings of the 9th European Workshop on White Dwarfs, ed. D. Koester \& K. Werner (Berlin: Springer-Verlag), 48

Jimenez, R., Jorgensen, U. G., Thejll, P., \& MacDonald, J. 1995, MNRAS, 275, 1245

Lawlor, T. M., \& MacDonald, J. 2002, Ap\&SS, 279, 123

Reimers, D. 1975, Mem. Roy. Soc. Sci. Liège, 8, 369

Ritossa, C., García-Berro, E., \& Iben, I. 1999, ApJ, 515, 381

Schönberner, D. 1979, A\&A, 79, 108

Schwarzschild, M., \& Härm, R. 1965, ApJ, 142, 855

Weiss, A. 1987, A\&A, 185, 165 